\title{
Health Monitoring System of Elderly using Wireless Sensor Network
}

\author{
Kothuru Anudeep \\ Dept. of ECE \\ Vardhaman College of Engineering \\ Hyderabad, India
}

\author{
S.Srinivas \\ Dept. of ECE \\ Vardhaman College of Engineering \\ Hyderabad,India
}

\begin{abstract}
Wireless-sensor-network-based home monitoring system for elderly activity behaviour involves functional assessment of daily activities. In this paper, we report a mechanism for estimation of elderly well-being condition based on usage of house-hold appliances connected through various sensing units. We define a two new wellness functions to determine the status of the elderly on performing essential daily activities. The modernized system for monitoring and evaluating the essential daily activities was tested at homes for four different elderly persons living alone and the results are encouraging in determining wellness of the elderly.
\end{abstract}

Keywords: Activities of daily living, elder care, home monitoring, smart home, wellness, wireless sensor network .

\section{INTRODUCTION}

WSN based health monitoring system for patient activities like body temperature, heartbeat ,blood pressure etc . By the using sensing units we can get the updates from the patient .An intelligent home monitoring system based on ZigBee wireless sensors network has been designed and developed to monitor and evaluate the patient details./Health conditions of an elderly people can be unsafe situations during regular works. Here is an software to get a health monitoring system to determine patient health care system.[4]Also, the system interprets all the essential elderly activities such as regular activities. Basically, the system function based on the usage data of electrical and non-electrical appliances within a home. At the hardware level, wireless sensor network with ZigBee [1] components are connected in the form of mesh topology, and a central coordinator of the sensing units collect data from the sensors connected to various appliances. In this system ,a required number of sensors for monitoring the daily activities of the elderly have been used. A smart sensor coordinator collects data from the sensing units and forward to the computer system for data processing. Collected sensor data are of low level information containing only status of the sensor as active or inactive and identity of the sensor. To sense the activity behavior of elderly in real time, the next level software module will analyze the collected data by following an intelligent mechanism at various level of data abstraction based on time and sequence behavior of sensor usage.

\subsection{OVER VIEW OF THE SYSTEM}

\subsubsection{Block diagram of Patient Section}

Above block diagram representing the patient section, in that we are continuously monitoring the patient information by using wireless sensor networks, i.e., Temperature and heart beat of patient. And that information we can forward to the control room section by using Zigbee technology[1].

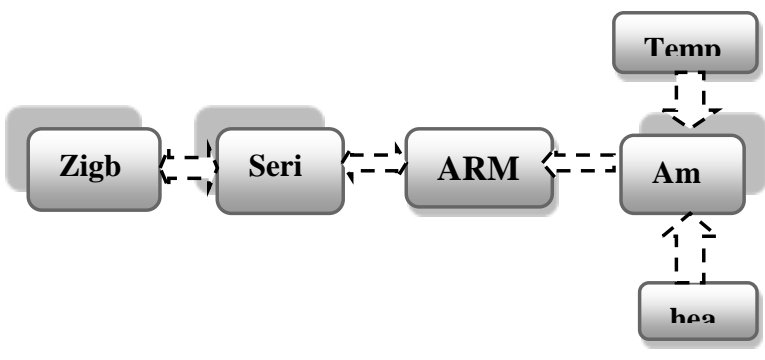

Figure 1. Patient Section

\subsubsection{Control Section}

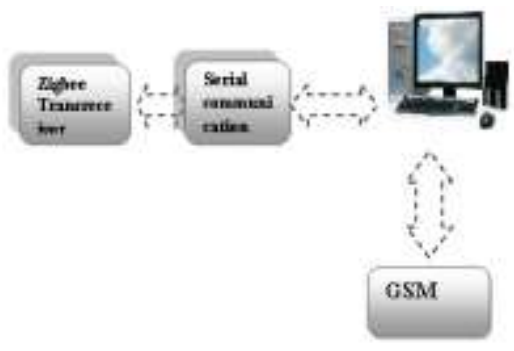

Figure 2. Control system

From patient section we transferring the information that is received by control room section through Zigbee and in pc it will check and if any abnormal condition occur it will send SMS to user by using GSM technology.

\subsubsection{Medical section}



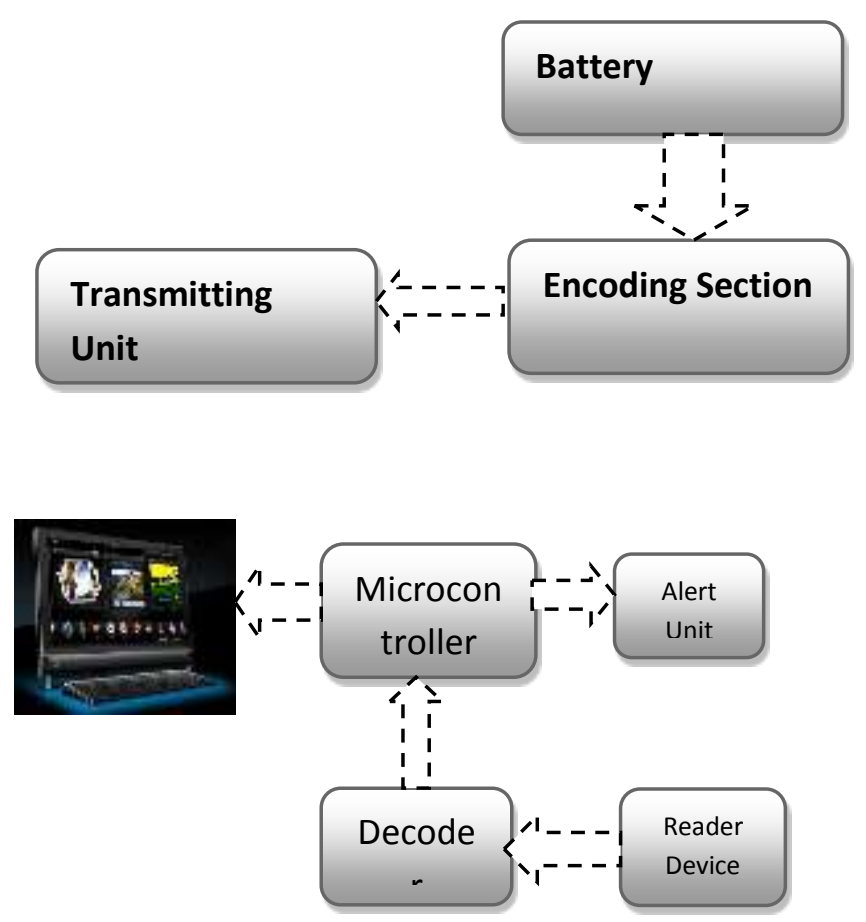

Figure 3. Medical Section

From the medical alert we are providing the patient information by using RF technology means, Person section consist RF transmitter followed Encoder. It transmitting the signal and the Medical section receiving the signal using RF receiver and if the person unauthorized means it will give the alert.

\subsection{Micro Controller (ARM7) Family}

The ARM7 family includes the ARM7TDMI-S, ARM7TDMI, ARM720T and ARM7EJ-S processors. The ARM7TDMI core is the most widely used 32-bit embedded RISC microprocessor solution. Optimized for power-sensitive applications and cost, the ARM7TDMI solution provides the low power consumption, high performance and small size needed in portable, embedded applications. The synthesizable version of the ARM7TDMI core, available in both VHDL and VERILOG, ready for compilation into processes supported by in-house or commercially available synthesis libraries. The ARM720T hard macro cell contains the ARM7TDMI core, Memory Management Unit (MMU), and a $8 \mathrm{~kb}$ unified cache that allows the use of protected execution spaces and virtual memory. This macro cell is compatible with leading operating systems including Windows CE, Linux, SYMBIAN OS, and palm OS.

The ARM7EJ-S processor comprises of ARM's latest DSP extensions and enabling acceleration of java-based applications. Compatible with the ARM9E'TM, ARM9TM, and ARM10 ${ }^{\mathrm{TM}}$ families, and Strong-Arm ${ }^{\circledR}$ architecture software written for the ARM7TDMI processor is $100 \%$ binarycompatible with other members of the ARM7 family and forwards-compatible with the ARM9E, ARM9 and ARM10 families, as well as products in Intel's Strong ARM and xscale architectures. This gives designers a software-compatible processor with strong price-performance points. Supporting the ARM architecture today includes:

$$
\text { - Operating systems such as Linux, }
$$

Windows CE, SYMBIAN OS and palm OS.

- More than 50 real-time operating systems including qnx and wind river's vx works

\subsection{LPC2148 Microcontroller}

LPC2148 Microcontroller Architecture. which offers very low power consumption and high performance . The ARM architecture is based on Reduced Instruction Set Computer (RISC) principles and the instruction set and related decode mechanism are much simpler than those of micro programmed Complex Instruction Set Computers (CISC). This simplicity results in a high instruction throughput and impressive real-time interrupt response from a small and costefficient processor core.

Pipeline techniques are employed so that all parts of the processing and memory systems can operate continuously. Typically, while one instruction is being executed, its successor is being decoded, and a third instruction is being fetched from memory. The ARM7TDMI-S processor also employs a unique architectural strategy known as Thumb, which makes it ideally suited to high-volume applications with memory restrictions, or applications where code density is an issue.

The key idea behind Thumb is that of a super-reduced instruction set. Essentially, the ARM7TDMI-S processor has two instruction sets:

- The standard 32-bit ARM set.

- A 16-bit Thumb set.

The Thumb set's 16-bit instruction length allows it to approach twice the density of standard ARM code while retaining most of the ARM's performance advantage over a traditional 16-bit processor using 16-bit registers. This is possible because Thumb code operates on the same 32-bit register set as ARM code. Thumb code is able to provide up to $65 \%$ of the code size of ARM, and $160 \%$ of the performance of an equivalent ARM processor connected to a 16-bit memory system.

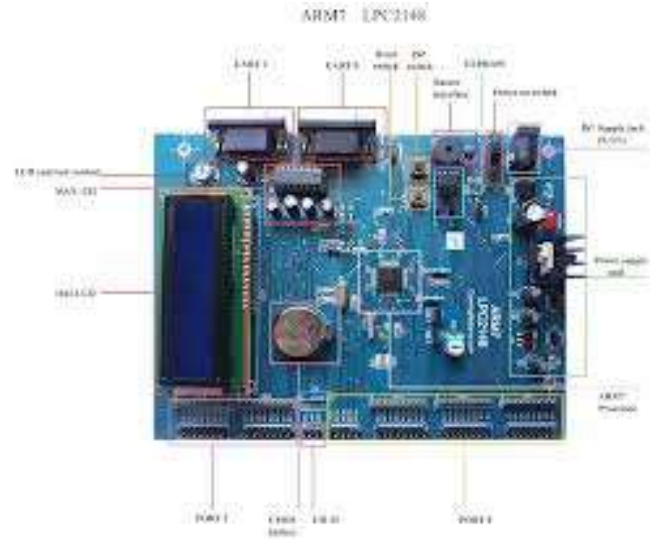

Figure 4. GSM Module

\subsection{Heart Beat Sensors}

The sensor consists of a light source and photo detector; light is shone through the tissues and variation in blood volume alters the amount of light falling on the detector. The source and detector can be mounted side by side to look at changes in reflected light or on either side of a finger or earlobe to detect 
changes in transmitted light. The particular arrangement here uses a wooden clothes peg to hold an infra red light emitting diode and a matched phototransistor. The infra red filter of the phototransistor reduces interference from fluorescent lights, which have a large AC component in their output

The skin may be illuminated with visible (red) or infrared LEDs using transmitted or reflected light for detection. The very small changes in reflectivity or in transmittance caused by the varying blood content of human tissue are almost invisible. Various noise sources may produce disturbance signals with amplitudes equal or even higher than the amplitude of [5] the pulse signal. Valid pulse measurement therefore requires extensive preprocessing of the raw signal. The setup described here uses a red LED for transmitted light illumination and a pin Photodiode as detector. With only slight changes in the preamplifier circuit the same hard- and software could be used with other illumination and detection concepts. The detectors photo current (AC Part) is converted to voltage and amplified by an inexpensive operational amplifier (LM358). A PIC16F877 microcontroller converts the analog signal with 10 bits resolution to a digital signal. An average is calculated from 250 readings taken over a 20 milliseconds period (This equals one period of the European power line frequency of $50 \mathrm{~Hz}$ ).

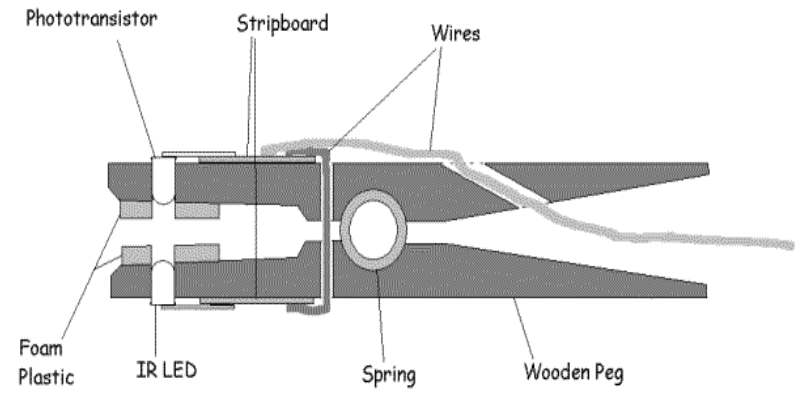

Figure 5. Heart Beat Sesnor

\subsection{Temperature Sensor}

The LM35 series are precision integrated-circuit temperature sensors, whose output voltage is linearly proportional to the Celsius (Centigrade) temperature. The LM35 thus has an advantage over linear temperature sensors calibrated in ${ }^{\circ}$ Kelvin, as the user is not required to subtract a large constant voltage from its output to obtain convenient Centigrade scaling. The LM35 does not require any external calibration or trimming to provide typical accuracies of $\pm 1 / 4^{\circ} \mathrm{C}$ at room temperature and $\pm 3 / 4^{\circ} \mathrm{C}$ over a full -55 to $+150^{\circ} \mathrm{C}$ temperature range. Low cost is assured by trimming and calibration at the wafer level. The LM35's low output impedance, linear output, and precise inherent calibration make interfacing to readout or control circuitry especially easy. It can be used with single power supplies, or with plus and minus supplies. As it draws only $60 \mu \mathrm{A}$ from its supply, it has very low self-heating, less than $0.1^{\circ} \mathrm{C}$ in still air. The LM35 is rated to operate over a $-55^{\circ}$ to $+150^{\circ} \mathrm{C}$ temperature range, while the $\mathrm{LM} 35 \mathrm{C}$ is rated for $\mathrm{a}-40^{\circ}$ to $+110^{\circ} \mathrm{C}$ range $\left(-10^{\circ}\right.$ with improved accuracy). The LM35 series is available packaged in hermetic TO-46 transistor packages, while the LM35C, LM35CA, and LM35D are also available in the plastic TO-92 transistor package. The LM35D is also available in an 8-lead surface mount small outline package and a plastic TO-220 package.

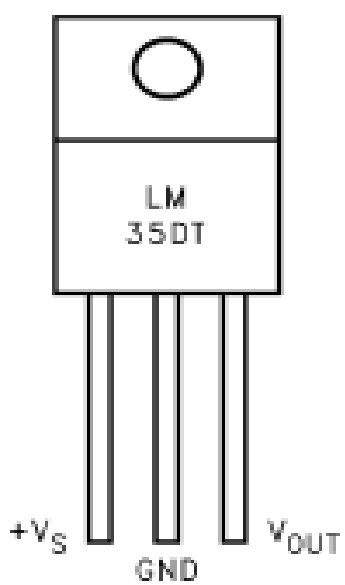

Figure 6. Temperature Sensor

\section{WIRELESS COMMUNICATION}

\subsection{GSM Technology}

To achieve important information of cars, one GSM Module is added into the car security system. Siemens TC35I GSM modem can quickly send SMS messages to appointed mobile phone or SMS server [3]. So the owner and the police can be informed at the first time. If another GPRS module is added in, the image data could also sent to information.

A GSM modem can be an external device or a PC Card or a PCMCIA Card. Typically, an external GSM modem is connected to a computer through a USB cable or a serial cable. A GSM modem in the form of a PC Card or a PCMCIA Card is designed for a laptop computer, which should be inserted into one of the PC Card or a PCMCIA Card slots of a laptop computer. A GSM modem needs a SIM card in order to operate. As mentioned in the earlier sections of the SMS tutorial, computers use a common set of standard AT commands to control the both the GSM and dial-up modems. GSM modem can be used just like a dial-up modem.

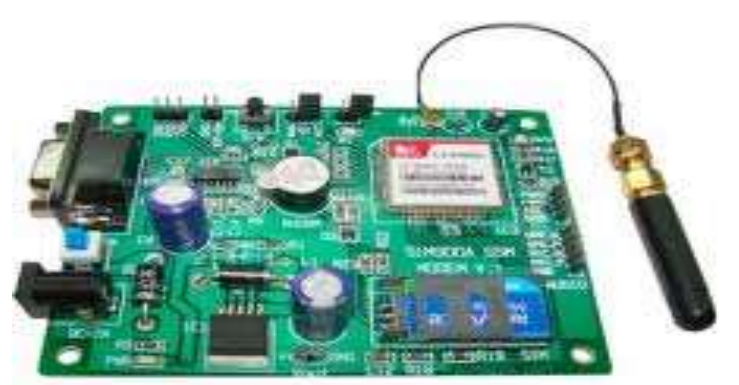

Figure 7. GSM Module 


\subsubsection{SMS commands}

- AT+CIMI

Note: Read the IMSI

- $\quad \mathrm{AT}+\mathrm{CMGS}=$ "+33146290800"

Note: Send a message in text mode

- $\quad$ AT+CMGR=3

Note: Read it

- $\quad \mathbf{A T}+\mathbf{C M G D}=3$

Note: Delete it Note: Message

\subsection{Zigbee Module}

The Xbee/Xbee-PRO RF Modules [1] are designed to operate within the ZigBee protocol and support the unique needs of low-cost, low-power wireless sensor networks. The modules require minimal power and provide reliable delivery of data between remote devices. The modules operate within the ISM 2.4 GHz frequency band and are compatible with the following:

- $\quad$ XBee RS-232 Adapter

- XBee RS-232 PH (Power Harvester) Adapter

- XBee RS-485 Adapter

- XBee Analog I/O Adapter

- XBee Digital I/O Adapter

- XBee Sensor Adapter

- XBee USB Adapter

- XStick

- $\quad$ Connect Port X Gateways

- 00 XBee Wall Router.

The XBee/XBee-PRO ZB firmware release can be installed on XBee modules. This firmware is compatible with the ZigBee 2007 specification, while the ZNet 2.5 firmware is based on Ember's proprietary "designed for ZigBee" mesh stack (EmberZNet 2.5). ZB and ZNet 2.5 firmware are similar in nature,(1) but not over-the-air compatible. Devices running ZNet 2.5 firmware cannot talk to devices running the ZB firmware.

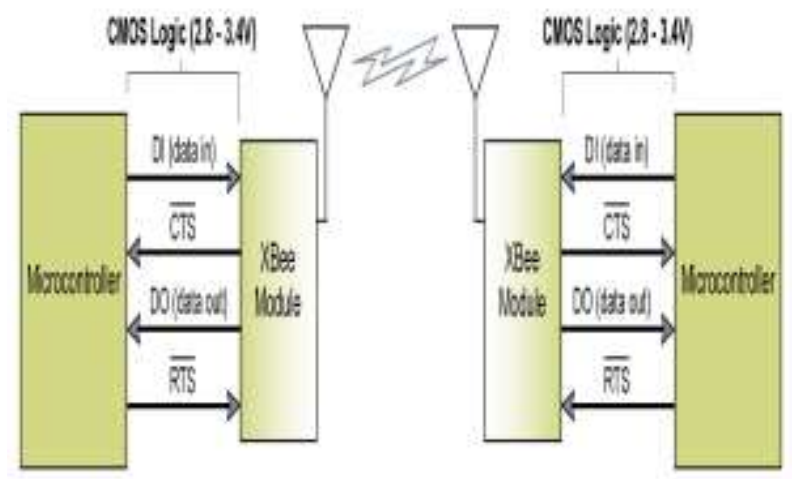

Figure 8. Zigbee Section

\section{MEDICAL SECTION}

\subsection{RF Transmitter and Receiver}

Radio Frequency, any frequency within the electromagnetic spectrum associated with radio wave propagation. When an
RF current is supplied to an antenna, an electromagnetic field is created that then is able to propagate through space. Many wireless technologies are based on RF field propagation Radio Frequency: The $10 \mathrm{kHz}$ to $300 \mathrm{GHz}$ frequency range that can be used for wireless communication. Also used generally to refer to the radio signal generated by the system transmitter, or to energy present from other sources that may be picked up by a wireless receiver(6).

\subsection{Transmitter}

The TWS-434 extremely small, and are excellent for applications requiring short-range RF remote controls(2). The transmitter module is only $1 / 3$ the size of a standard postage stamp, and can easily be placed inside a small plastic enclosure.

TWS-434: The transmitter output is up to $8 \mathrm{~mW}$ at $433.92 \mathrm{MHz}$ with a range of approximately 400 foot (open area) outdoors. Indoors, the range is approximately 200 foot, and will go through most walls.

\subsubsection{1 (c)Receiver}

3.2.1.2 RWS-434: The receiver also operates at 433.92MHz, and has a sensitivity of $3 \mathrm{uV}$. The WS-434 receiver operates from 4.5 to 5.5 volts-DC, and has both linear and digital outputs.

\subsubsection{3 (d)Transmitting and receiving}

Full duplex or simultaneous two-way operation is not possible with these modules. If transmit and receive module are in close proximity and data is sent to a remote receive module(2) while attempting to simultaneously receive data from a remote transmit module, the receiver will be overloaded by its close proximity transmitter. This will happen even if encoders and decoders are used with different address settings for each transmitter and receiver pair. If two way communications is required, only half duplex operation is allowed.(6)

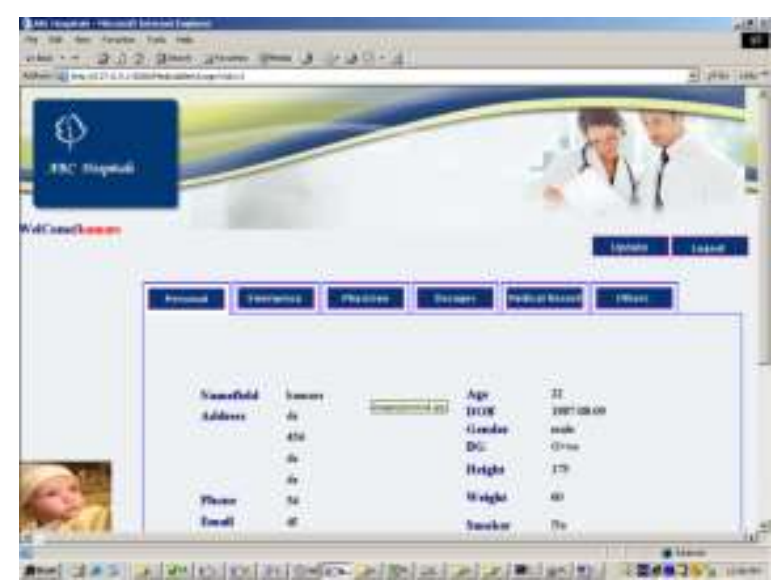




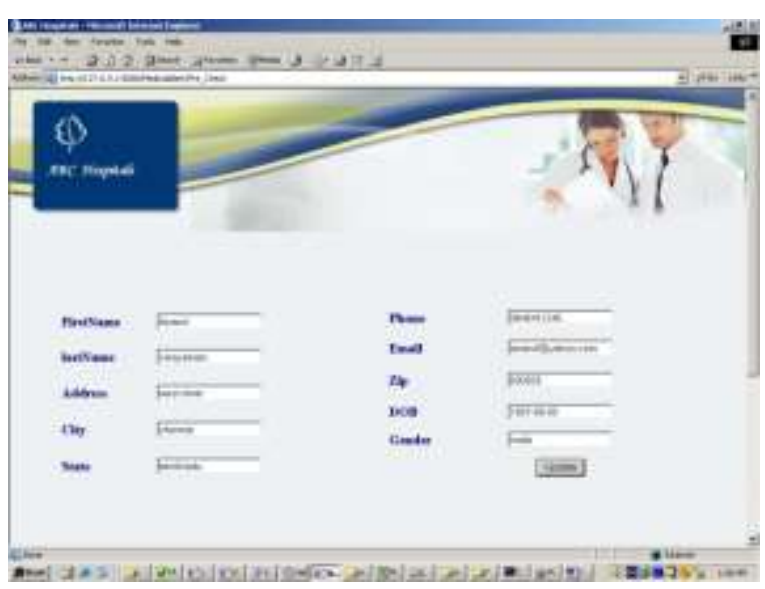

Figure 9. Screens for Medical Data Storing

\section{CONCLUSION}

In this time, model biotelemetry system is being implemented into working solution. Nevertheless, there is space for improvements in both concept and implementation details of this system. Model biotelemetry system is currently designed for indoor use by one patient only. More nearby instances of inner part of model biotelemetry system managed by single outer part of system are possible, but there exists one to one mapping between patient and ZigBee network [1]. Future improvements may include support for outdoor operation with communication implemented using 3G mobile technology [3] and patient's tracking by GPS system. With advancements in low-power high-density FPGA solutions, FPGA programmable system on chip technology seems to be promising for purpose of this biotelemetry system.

\section{REFERENCES}

[1] Safaric S., Malaric K., "ZigBee wireless standard",Multimedia Signal Processing and Communications, $48^{\text {th }}$ International Symposium ELMAR-2006, Zadar, Croatia,June 2006.

[2] Ze Zhao and Li Cui, "EasiMed: A remote health care solution", Proceeding of the 2005 IEEE Engineering in Medicine and Biology 27th Annual Conference, Shanghai,China, September 2005.

[3] Krejcar, O., Janckulik, D., Motalova, L., Kufel, J., "Mobile Monitoring Stations and Web Visualization of Biotelemetric System - Guardian II". In EuropeComm 2009. LNICST vol. 16, pp. 284-291. R. Mehmood, et al. (Eds). Springer, Heidelberg (2009).

[4] Krejcar, O., Janckulik, D., Motalova, L., "Complex Biomedical System with Mobile Clients". In The World Congress on Medical Physics and Biomedical Engineering 2009, WC 2009, September 07-12, 2009 Munich, Germany. IFMBE Proceedings, Vol. 25/5. O.Dössel, W. C. Schlegel, (Eds.). Springer, Heidelberg. (2009).

[5] Krejcar, O., Janckulik, D., Motalova, L., Frischer, R., "Architecture of Mobile and Desktop Stations for Noninvasive Continuous Blood Pressure Measurement".

In The World Congress on Medical Physics and Biomedical Engineering 2009, WC 2009, September 0712, 2009 Munich, Germany. IFMBE Proceedings, Vol. 25/5. O. Dössel, W. C. Schlegel, (Eds.). Springer, Heidelberg. (2009).

[6] Idzkowski A., Walendziuk W.: Evaluation of the static posturograph platform accuracy, Journal of Vibroengineering, Volume 11, Issue 3, 2009, pp.511516, ISSN 1392 - 8716M. Penhaker, M. Cerny, L. Martinak, et al. HomeCare "Smart embedded biotelemetry system" Book Series IFMBE proceedings World Congress on Medical Physics and Biomedical Engineering, AUG 27-SEP 01, 2006 Seoul, SOUTH KOREA, Volume: 14 Pages: 711-714, 2007, ISSN: 1680- 0737, ISBN: 978-3-540-36839-7. 